\title{
Training in basic hand-held echocardiography for critical care: not such a difficult task
}

\author{
Z Belamaric, D Nagore, JP Jimeno, A Bilbao, T Garth, K Plummer \\ Department of Perioperative Medicine, St. Bartholomew's Hospital, London, UK
}

Background: In the last decade focus assessment ultrasound protocols (FAST, FATE, RUSH,...) have been developed. Due to technological development simple scans can be delivered by palm top devices. This study is designed to see if the skills needed to provide basic hand-held echocardiography in the critical care environment can be taught effectively

Method: 12 echocardiography naive junior doctors received a single day course, consisting of lectures, simulation work and familiarisation with the hand held $V$ scanners. They had access to the $V$ scanners and $a$ mentor for a 6 week period. After this period they were evaluated to assess knowledge and skills learnt and retained jointly by a senior ICU anaesthetist and a senior cardiologist. The following categories were assessed and classified in a scale of 1 to 5 , where (1) poor, (2) fair, (3) good, (4) very good and (5) excellent : 1 . Image acquisition of the standard ECHO views; 2. LV assessment of size and function; 3. RV function assessment; 4. MV and MR assessment; 5. Aortic Valve assessment and to identify AS, AR and valve thickening; 6. Pericardial effusion assessment; 7. Filling status assessment including IVC measurement 8. Overall Impression of the junior doctor knowledge

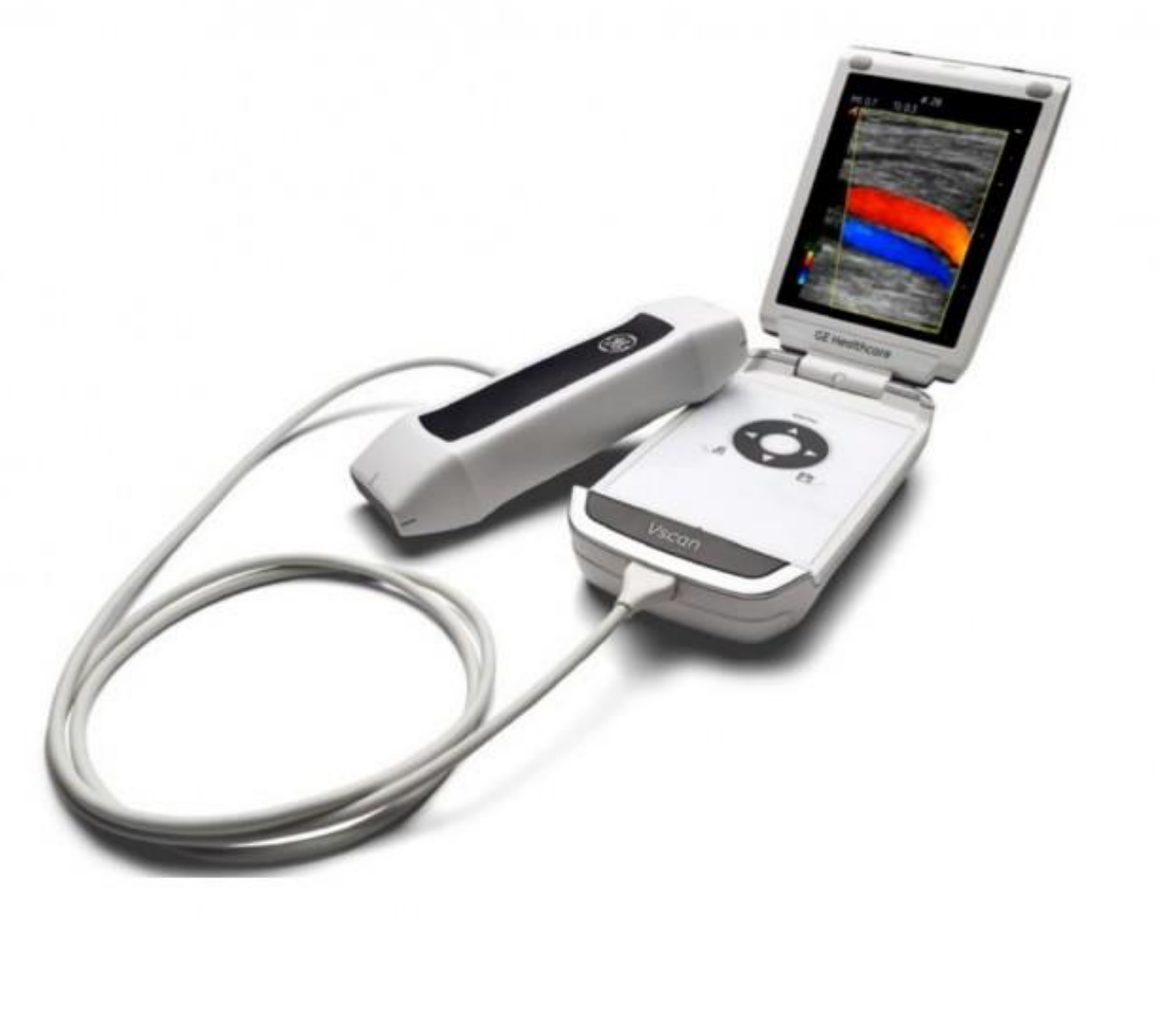

\section{Comparison between mean competency scores pre- and post-training}

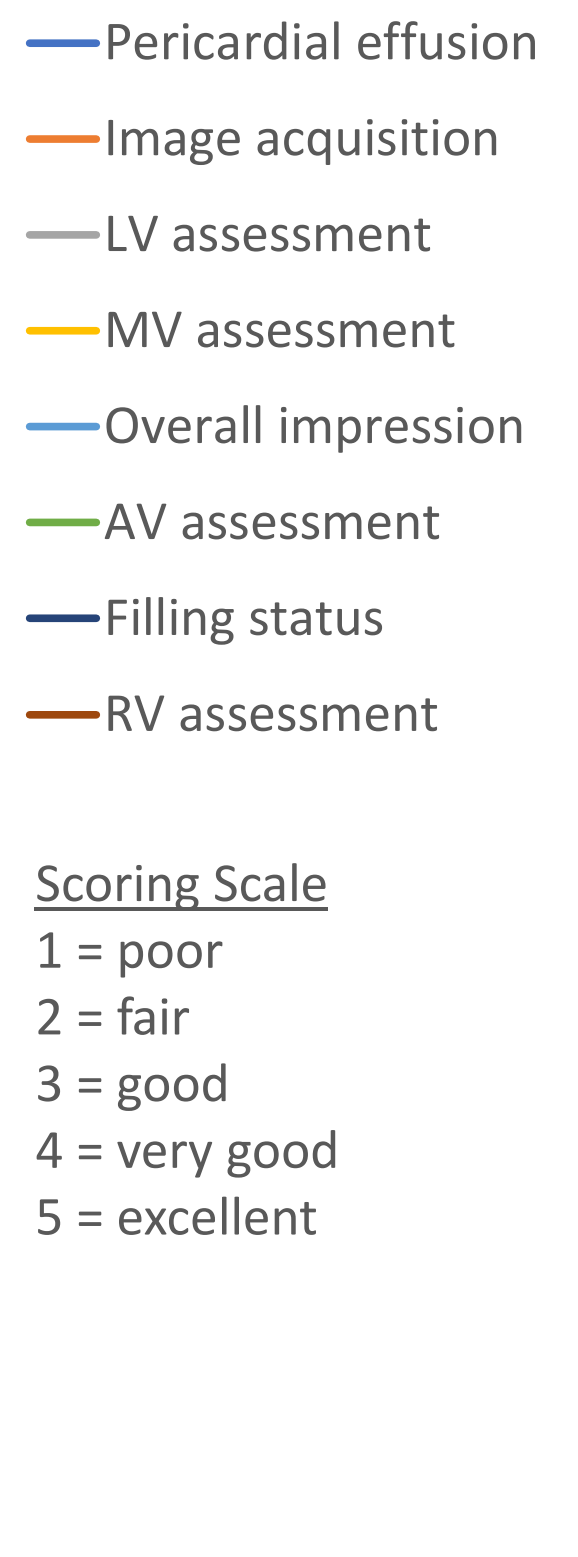

\section{Conclusion}

Junior doctors have significantly improved overall knowledge in the basic echocardiography after the course. The level of knowledge was equally distributed in all categories except in RV and filling status assessment where students showed lower skills level. Therefore, appropriate clinical and imaging supervisory pathways should always be available where this kind of activity is undertaken. 